\title{
Infectious Disease Management and Control with Povidone Iodine
}

\author{
Maren Eggers
}

Received: June 6, 2019 / Published online: August 14, 2019

(c) The Author(s) 2019

\begin{abstract}
With reports of vancomycin-resistant enterococci recently emerging in hospital settings, renewed focus is turning to the importance of multifaceted infection prevention efforts. Careful compliance with established hygiene practices by healthcare workers together with effective antiseptic options is essential for the protection of patients from infectious agents. For over 60 years, povidone iodine (PVP-I) formulations have been shown to limit the impact and spread of infectious diseases with potent antiviral, antibacterial and antifungal effects. In addition to a lack of reported resistance, the benefits of PVP-I include an excellent safety profile and a broad spectrum of effect due to its multimodal action. Studies have shown that hand washing with PVP-I-based antiseptics is effective for the decontamination of skin, while PVP-I mouthwashes and gargles significantly reduce viral load in the oral cavity and the oropharynx. The importance of PVP-I has been emphasised by its inclusion in the World Health Organization's list of essential medicines, and
\end{abstract}

Enhanced digital features To view enhanced digital features for this article go to https://doi.org/10.6084/ m9.figshare.9168032.

M. Eggers $(\bowtie)$

Labor Prof Gisela Enders MVZ GbR, Stuttgart, Germany

e-mail: eggers@labor-enders.de high potency for virucidal activity has been observed against viruses of significant global concern, including hepatitis A and influenza, as well as the Middle-East Respiratory Syndrome and Sudden Acute Respiratory Syndrome coronaviruses. Together with its diverse applications in antimicrobial control, broad accessibility across the globe, and outstanding safety and tolerability profile, PVP-I offers an affordable, potent, and widely available antiseptic option.

Funding Mundipharma Singapore Holding Pte Limited.

Keywords: Infection control; Infectious disease; Povidone iodine; Sterility; Viral outbreak
Abbreviations
HFMD Hand, foot, and mouth disease
HIV Human immunovirus
ICU Intensive care unit
MDCK Madin-Darby canine kidney
MERS Middle East Respiratory Syndrome
MRSA Methicillin-resistant Staphylococcus aureus
PVP-I Povidone iodine
SARS Severe Acute Respiratory Syndrome
UV Ultraviolet
WHO World Health Organization 


\section{BACKGROUND}

The importance of robust infection prevention practices has been highlighted recently with reports of vancomycin-resistant enterococci (VRE) emerging in hospitals in Switzerland [1], Australia [2] and New Zealand [3]. Recently, an Australian study has shown that Enterococci faecium, the microorganism responsible for various nosocomial infections, was behind over $30 \%$ of enterococcal bacteremia cases surveyed in the country [4]. It was found that $90 \%$ of these were ampicillin-resistant CC17 strains, while 50\% were also vancomycin-resistant. Other evidence suggests that some E. faecium strains may be expressing limited resistance to alcohol-based sanitisers, although at concentrations significantly lower than is recommended for use in hospital settings [5]. The continuing spread of antibiotic resistance is an area of significant concern and necessitates the careful consideration of more rigorous management practices, as well as the assessment of alternative antiseptics for broad hygiene applications.

Povidone iodine (PVP-I) is a widely-available alternative antiseptic to alcohol that is commonly used in clinical settings, including for skin disinfection before and after surgery. It is usually applied to the skin as a liquid or a powder and can be used to treat current infections and prevent the spread of opportunistic pathogens. PVP-I has a broad antimicrobial spectrum and is active against a plethora of viruses and antibiotic-resistant bacterial strains (Table 1). Susceptible Gram-negative bacteria include Klebsiella pneumoniae, a common pathogen in hospitals, and susceptible Grampositive bacteria include methicillin-resistant Staphylococcus aureus and Escherichia coli. In addition, PVP-I has been shown to be superior to chlorhexidine in hand washing studies comparing efficacy against bacteria and viruses (Table 2).

PVP-I formulations first became available in 1955 , and the active ingredient is listed on the World Health Organization's (WHO) List of Essential Medicines, a list of the most important medicines necessary for any functional healthcare system. PVP-I is available over the counter and is often used as a broad-spectrum topical antiseptic treatment for minor cuts, burns, and abrasions, as well as in surgical operating theatres. Widespread use in diverse clinical and non-clinical settings over recent decades has made the numerous advantages of PVP-I formulations more apparent. In addition to a broad-spectrum effect and excellent safety profile, active iodine has various properties that can aid in wound healing, with a strong evidencebased rationale existing for the application of PVP-I in treating infected wounds. The European Wound Management Association has published a position paper that acknowledges the broad spectrum of PVP-I activity against bacteria, viruses, fungi and endospores [6]. In contrast to other antiseptics, significant resistance or cross-resistance has not been observed for iodine, likely due to the various mechanisms through which iodine elicits its effects. It has thus found diverse applications in healthcare as a sterilising agent for pre- and post-operative

Table 1 Comparison of antimicrobial activities of common antiseptic classes $[12,13]$

\begin{tabular}{lllll}
\hline Antiseptic type & Inactivates & & \\
\cline { 2 - 5 } & Bacteria & Bacterial spores & Enveloped viruses & Non-enveloped viruses \\
\hline Quaternary ammonium & + & + & - & - \\
Chlorine & + & Variable & + & + \\
Ethanol & + & - & + & Variable \\
Iodine & + & + & + & + \\
Phenolic & + & - & + & Variable \\
\hline
\end{tabular}


Table 2 Data from direct comparisons of antimicrobial activities of common antiseptics in hand washing studies [14, 15]

\begin{tabular}{llll}
\hline Antiseptic type & Bacterial CFUs & Escherichia coli & Murine norovirus \\
\hline Chlorhexidine & ++ & +++ & + \\
Povidone-iodine & +++ & +++ & +++ \\
$n$-Propanol & +++ & Not compared & Not compared \\
\hline
\end{tabular}

CFUs Colony-forming units

skin cleaning, for the prevention and treatment of infections in ulcers, and in many other applications. The formulations are typically manufactured with concentrations of $7.5-10 \%$ PVP-I in solution, with oral formulations including $1 \%$ oral gargle, in addition to surgical scrubs, ointments, and swabs.

Preclinical and clinical safety and efficacy data show that PVP-I exhibits characteristics that are well-suited to wound-healing scenarios, including efficacy against biofilms, good tolerability and some degree of anti-inflammatory effect $[7,8]$. Of clinical relevance, healing in clean wounds is not suppressed by PVP-I, while it is supported in wounds that are colonised by microorganisms. It is particularly useful for the treatment of sensitive wounds and those where an extended duration of therapy is needed. Medicated gauzes are now widely available, making the formulations easier to apply [9].

PVP-I also elicits potent antiviral activity, with commercially available formulations shown to be more effective in terms of viral reduction than alcoholic and non-alcoholic sanitisers, as well as antimicrobial soaps [10]. Alcohol is a widely used antiseptic likely due to its affordability and relative ease of manufacture, but has been shown to be less effective than PVP-I at killing microorganisms [11].

Compliance with Ethics Guidelines This article is based on previously conducted studies and does not contain any studies with human participants or animals performed by any of the authors.

\section{MECHANISMS OF ACTION OF PVP-I}

PVP-1 refers to an iodine preparation commonly used in both household and healthcare settings. It consists of a complex of povidone, hydrogen iodide, and elemental iodine which targets structures critical to the survival and replication of microorganisms. Common formulations typically consist of a 10\% PVP-I solution containing $1 \%$ available iodine.

Following application, elemental iodine can take on several forms in aqueous solution, with the molecular $I_{2}$ and hypoiodous acid (HOI) being the most effective in terms of antimicrobial activity [16]. The iodine molecules are free to oxidise vital pathogen structures such as amino acids, nucleic acids and membrane components. An equilibrium is achieved in such circumstances, with more PVP-bound iodine released into solution to replace the iodine that is consumed by germicidal activity. The maintenance of this equilibrium ensures long-lasting efficacy during bouts of microorganism proliferation, as well as better tolerability for patients due to lower levels of irritation. Electron microscopy and biochemical observations support the hypothesis that PVP-I disrupts microbial cell walls by inducing pore formation, leading to cytosol leakage [17]. The lack of reported resistance to PVP-I to date is thought to be due to the sheer diversity of susceptible targets within each pathogen, an important aspect to be considered in the face of rising concerns for antibiotic resistance. 


\section{PVP-I AGAINST ANTIMICROBIAL- RESISTANT BACTERIAL STRAINS}

The emergence of antibiotic resistant strains of bacteria, including VRE and methicillin-resistant Staphylococcus aureus (MRSA), has become a significant issue for healthcare facilities throughout the world. Indeed, studies have shown that approximately $42 \%$ of $S$. aureus isolates in Europe and Japan harbour genes that enable resistance to quaternary ammonium compounds and chlorhexidine, with chlorhexidine overuse thought to be a factor in emerging resistance in some strains of Gram-negative bacteria $[18,19]$. The prevalence of methicillin resistance harboured by strains of $S$. aureus capable of causing bloodstream infection between 1990 and the early 2000s in the UK rose significantly from 2 to $>40 \%$, with mean overall rates of bacteraemia involving MRSA ranging from 0.10 to 0.19 per 1000 occupied beds [7]. The overuse of antibiotics is thought to be a contributing factor towards rising antibiotic resistance, and is now being discouraged in favour of the wider usage of antiseptics, to which it is more difficult for bacteria to develop resistance [7].

While evidence of cross-resistance to antiseptics and antibiotics has been documented for many agents, in over a century of use, no significant acquired resistance or crossresistance has been reported for iodine when used for specific indications [20]. This striking lack of resistance is thought to be due to the diverse mechanisms through which iodine simultaneously exerts its effects. Although some aspects remain to be fully understood, iodine's microbicidal activity is known to involve the oxidation of bacterial cell components, including amino/fatty acids, nucleotides, lipids in the cell membrane, and enzymes in the cytosol, ultimately promoting their denaturation and deactivation [16]. More specifically, the multimodal action of iodine is known to arise from the potent oxidation of $\mathrm{NH}_{-}, \mathrm{OH}_{-}$, and $\mathrm{SH}-$ groups on amino acids, nucleotides and unsaturated fatty acids, and the emergence of resistance is likely to be prevented by the sheer diversity of targets affected. Such effects eventually result in the simultaneous inactivation of bacterial enzymes, a loss of genome integrity, and cell wall damage, overwhelming the microorganism's repair mechanisms.

In a hallmark study, the development of bacterial resistance to iodine was investigated by serial passage of two strains of Pseudomonas aeruginosa, two strains of Escherichia coli, two strains of Klebsiella aerogenes, and one strain of Serratia marcescens in the presence of sub-optimal concentrations of iodine that were insufficient to cause cell death [21]. The investigators found that, after 20 passages, no detectable change was observed in the minimal inhibitory concentration of iodine needed, nor the time taken until cell death occurred between the parent strain and the passaged subcultures when exposed to efficacious concentrations of iodine. The PVP-I formulation containing up to $1 \%$ available iodine was able to kill all strains tested in under $5 \mathrm{~min}$, with most cells being destroyed within $30 \mathrm{~s}$. While dilute concentrations were noted to take in excess of $10 \mathrm{~min}$ to achieve an effect, even these iodine dilutions were successful in killing all strains upon prolonged exposure. In real-world scenarios, over-the-counter PVP-I formulations are accommodating of such prolonged exposure to healthy skin, with some commercial formulations known to be active for $12-14 \mathrm{~h}$, compared to the $1-4 \mathrm{~h}$ of activity documented for chlorhexidine against fungi and endospores. Of particular note, clinical isolates of chlorhexidine-resistant Klebsiella pneumoniae that are also cross-resistant to colistin have recently been identified [22]. While chlorhexidine is commonly used in disinfectants, these new findings suggest that exposure to chlorhexidine is associated with stable resistance to colistin, an antibiotic of last resort for multidrug-resistant infections.

\section{PVP-I IN ANTIVIRAL APPLICATIONS}

Various experimental models have been developed to examine the antiviral properties of particular agents, with testing recommended to be taken in a stepwise approach. The European Committee of Standardization (CEN) 
recommends that the first phase involve an in vitro suspension test with enveloped viruses (representing the bulk of emerging infectious disease threats). Phase 2 involves similar conditions, but with non-enveloped viruses, while the third and final phase involves human hands in a simulation study. In addition to a virucidal hand test, variations on the latter step can include a quantitative non-porous surface test without mechanical action, a quantitative carrier test, and a so-called 4-field test involving surface disinfection with mechanical action (Fig. 1).

Similarly, the US Center for Disease Control recommends a standardised method simulating hand washing with the antiseptic formula to be tested to determine efficacy in reducing hand microflora [23]. New test models have enabled the assessment of PVP-I formulations against highly infective and dangerous pathogens that may not be possible to test in vivo due to clinical risks and ethical considerations. Various commercially available formulations have achieved the standards of the latest European guidelines (EN 14476 and EN 1499), with efficacy demonstrated in both the Enveloped Virus Test Model (MVA) and Non-Enveloped Virus
Test Model (MNV). The use of such model viruses can provide valuable data for informed decision-making during public health crises.

EN 14476 is a standardised inactivation assay that involves a virus suspension, an interfering substance (such as bovine serum albumin), and the substance to be tested [24]. A virus control mixture is used to compare the effects of the antiviral product following a specified contact time (e.g. 15, 30 or 60 s), with virucidal activity calculated by determining the difference in logarithmic titre between the virus control and the test virus cultures.

The assessment of microbicidal efficacy can be challenging, due to the difficulties in direct observation and the sheer numbers of cells or particles involved. Consensus within the medical community has settled upon a minimum measure required to evaluate microbicidal efficacy, referred to as the $\log _{10}$ reduction factor. This is a mathematical term measured by titration at the endpoint and indicates the reduction in the number of living or viable microbes after treatments such as sanitisation, disinfection, or cleaning. European Standards (EN) stipulate a minimum level of $\geq 4 \log _{10}$ reduction in titre for viruses and fungi, and $a \geq 5 \log _{10}$ reduction

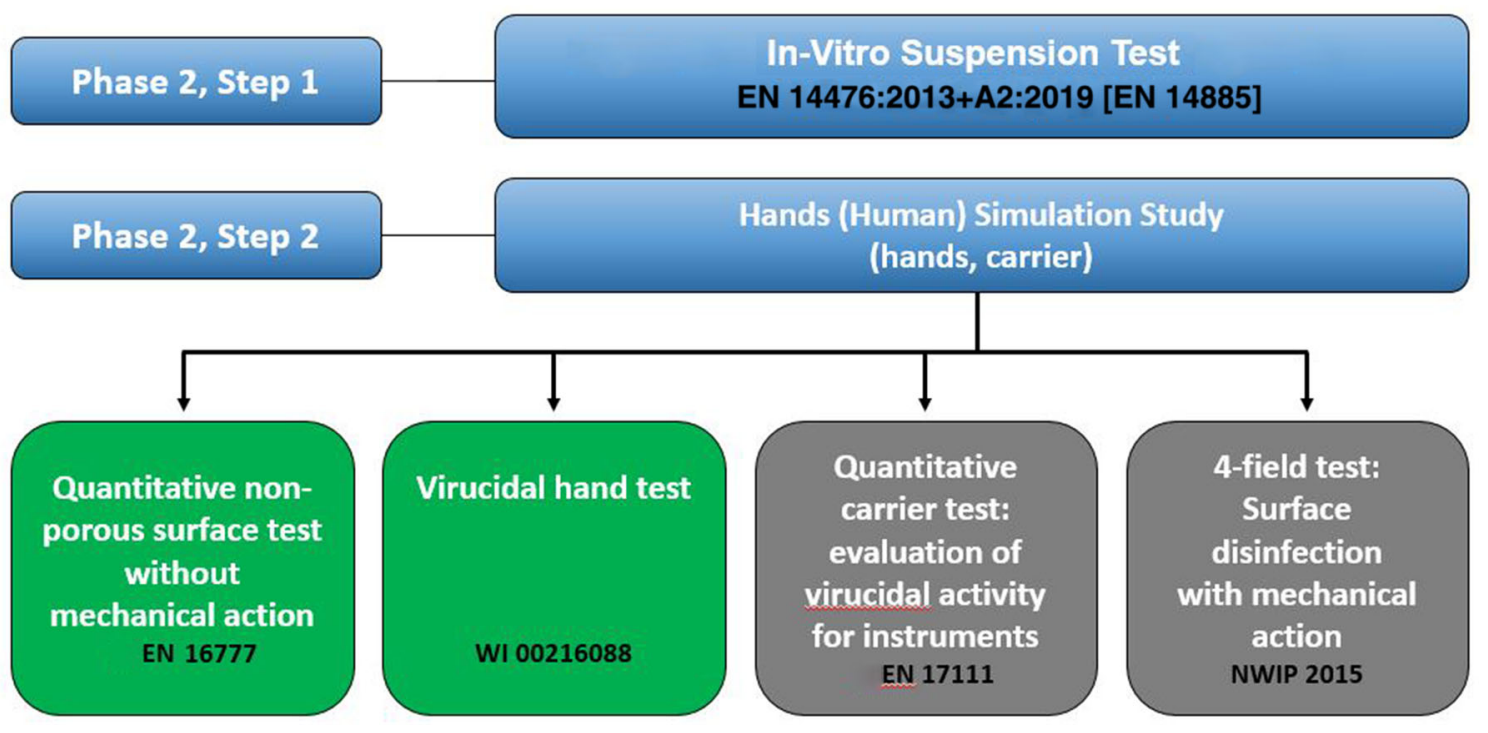

Fig. 1 Stepwise approach according to the European Committee of Standardization (CEN). Hand disinfection tests highlighted in green. Stepwise approach as proposed at

the 2nd International Meeting on Respiratory Pathogens (IMRP) held in Singapore on March 9, 2018 (see: https:// www.isirv.org) 
for bacteria, representing reductions in the absolute number of microbes by $99.99 \%$ and 99.999\%, respectively (Table 3).

The introduction and use of model viruses has significantly aided in the investigation of new anti-virucidal agents, particularly during times of pressing need. For example, in December 2013, the Ebola virus was first discovered in Guinea, and rapidly became one of the most complex epidemics in recent history. Due to its high biosecurity level, research into vaccines and containment measures for the virus was highly limited. Although yet to be confirmed as a surrogate for Ebola virus, the modified vaccinia virus (MVA) was introduced in 2014 with a reference claim against "enveloped viruses for hygienic hand rub and hand wash" [10]. Such models allow for reasonable progress to be made in comparing antiviral agents in certain settings. Similarly, Middle East Respiratory Syndrome (MERS) was first discovered in 2012, with the virus now having infected more than 1300 victims in 26 countries, resulting in more than 480 deaths. Transmission is known to frequently occur in healthcare settings, highlighting the need for suitable models to test containment measures. The modified vaccinia virus, Ankara, has been used as a test model for MERS, with similar structural features and cultivation measures [24].

The influenza virus has been responsible for some of the most significant epidemics in the modern world, with annual outbreaks resulting in approximately 3-5 million cases of severe illness and between 250,000 and 500,000 deaths per year [25]. An influenza study using plaque inhibition assays showed that a $1.56-\mathrm{mg} / \mathrm{ml}$ PVP-I treatment can inhibit infections in MDCK cells by human (eight strains) and avian (five strains) influenza A viruses, including H1N1, H3N2, H5N3 and H9N2, from 23 to 98\%. Receptor binding analysis revealed that haemagglutinin inhibition was the likely cause of the PVP-I virucidal activity, rather than the inhibition of host-specific sialic acid receptors. The finding also demonstrates two specific mechanisms of reduction of viral growth, namely, PVP-I blockade of viral attachment to the host cell receptors and the inhibition of viral release from infected cells [26].

PVP-I formulations are also known to have broad antiviral properties. These effects are mechanistically similar in principle to iodine's antibacterial activity. For example, the virucidal mechanisms of action of PVP-I have been determined to involve the inhibition of essential viral enzymes such as neuraminidase. The inactivation of this enzyme blocks viral release from the host cell, preventing further spread of the virus to uninfected cells. In addition, PVP-I also inhibits viral haemagglutinin, resulting in the blockade of attachment to host cell receptors. By simultaneously targeting both critical aspects of the viral machinery needed for replication, PVP-I reduces the likelihood of resistance emerging through sudden mutation.

Under such guidelines, PVP-I formulations have been shown to elicit viral inactivation of $>99.99 \%$ in test systems using a modified vaccinia virus [24]. Virucidal efficacy has in some cases been determined to occur within $15 \mathrm{~s}$ of contact. Following a hand simulation study with the murine norovirus, it was found that hand washing with PVP-I was more effective than chlorhexidine and soft soap, a gold standard recommended by the WHO. PVP-I was

Table $3 \log _{10}$ reduction factor: the minimum measure of microbicidal efficacy [EN 14885]

\begin{tabular}{llll}
\hline & Virus & Bacteria & Fungi/yeast \\
\hline $\begin{array}{l}\log _{10} \text { reduction to achieve (reduction in } \\
\text { microbial titre) }\end{array}$ & $\geq 4$ & $\geq 5$ & $\geq 4$ \\
$\begin{array}{l}\text { Microbial reduction (\%) } \\
\text { Standard }\end{array}$ & $99.99 \%$ & $99.999 \%$ & $99.99 \%$ \\
& $\begin{array}{c}\text { EU Standards (EN) German } \\
\text { Guidelines }\end{array}$ & $\begin{array}{c}\text { EU Standards } \\
\text { (EN) }\end{array}$ & $\begin{array}{c}\text { EU Standards } \\
\text { (EN) }\end{array}$ \\
\hline
\end{tabular}


also shown to be more virucidal against both enterovirus and coxsackievirus when compared to other disinfectants.

The need to develop potent antiviral formulations suitable for widespread use has been brought to prominence by the emergence of rapid viral outbreaks over the past decade, many of which have been coronaviruses. The Middle East Respiratory Syndrome coronavirus (MERS$\mathrm{CoV}$ ) is a single-stranded RNA virus first identified during an outbreak in 2012 that eventually spread to 21 countries worldwide, triggering mass media coverage [24]. To date, the virus remains categorised as a high biosafety risk, with containment remaining the primary measure to combat outbreaks, as no vaccines or specific antiviral treatments have yet been developed. However, randomised controlled clinical trials have shown that PVP-I and alcohol-based hand rubs are more effective than soap-based hand washes for hand hygiene in the presence of such transmissible viruses [16].

In a study evaluating mouthwash, surgical scrubs, and skin cleanser formulations of PVP-I for antiviral activity against the MERS coronavirus, it was shown that the viral titre could be reduced by a factor of $\mathrm{C} 4 \log _{10}$, corresponding to a c.99.99\% inactivation level [24]. This remarkable level of potency was achieved within $15 \mathrm{~s}$ of application of each PVP-I formulation, which included a 7.5\% PVP-I surgical scrub, a 1\% PVP-I gargle/mouthwash and a $4 \%$ PVP-I skin cleanser formulation under the brand name Betadine (Mundipharma, Limburg, Germany). The findings indicate that PVP-Ibased hand hygiene products can be used to decontaminate virally-infected skin, while PVPI mouthwash can reduce viral load in the oral cavity and the oropharynx, potentially aiding in the support of hygiene measures needed to reduce the severity of future MERS outbreaks.

An earlier cooperative study presenting results in comparison with other antiseptics has shown how PVP-I impacts the infectivity of some of the most significant human pathogenic viruses, including polio-, HIV-1, adeno-, rota-, mumps, rhino-, coxsackie-, rubella, herpes-, measles, and influenza viruses. Mumps and adeno-viruses have been decimated in test settings by a more than 3-log reduction within $60 \mathrm{~s}$ of exposure to PVP-I concentrations higher than $0.5 \%$ [27]. Influenza virus was inactivated by a more than 5-log reduction following $15 \mathrm{~s}$ at the same dose levels, while HIV was reduced by a more than $4.5-\log$ reduction after a 30-s exposure to doses higher than $0.05 \%$. However, coxsackievirus and poliovirus type 1 were observed to be not as sensitive to PVP-I inactivation, with both viruses requiring doses higher than $0.125 \%$ for inactivation, as was the case for rhinovirus. The authors concluded that PVP-I preparations were effective against measles, mumps, herpes, HIV, influenza, and rota-viruses, while rubella, polio-, adeno-, and rhinoviruses were only sensitive to higher doses. The fact that both virus types could be either sensitive or resistant regardless of whether they were enveloped or non-enveloped suggests that mechanisms specific to certain viral types are likely to some extent to influence iodine sensitivity. Overall, the findings are of particular relevance given that an overwhelming proportion of sore throat cases are thought to be of viral origin, and there appears to remain an overprescribing of antibiotics in such cases.

\section{PVP-I FOR HOSPITAL INFECTION CONTROL}

Hospital settings are particularly challenging for antisepsis, as antibiotic-resistant strains are a constant threat. PVP-I is widely used in surgical settings to prevent infection by ensuring preoperative decontamination. The aim of such decontamination is to reduce the risk of skin flora being introduced into sensitive areas once the skin barrier has been breached. Normal and innocuous bacterial flora that usually colonise healthy skin can become harmful in such settings, particularly for immuno-compromised individuals. A PVP-I surgical scrub with a detergent and foam booster is recommended for the most effective preoperative sterilisation [11].

A comprehensive literature review by a joint committee consisting of representatives from the British Society of Antimicrobial Chemotherapy, the Hospital Infection Society, and the Infection Control Nurses Association 
concluded that $7.5 \%$ povidone iodine or $2 \%$ triclosan is helpful for the eradication and suppression of skin colonisation for short periods, particularly in preoperative settings [19]. The Working Party recommended in its findings that patients bathe daily for 5 days with an appropriate antiseptic detergent. The skin should be moistened, and an antiseptic detergent should be applied thoroughly to all areas before rinsing in the bath or shower. The use of such antiseptics was also recommended for all other washing procedures and for bed bathing.

Patients requiring endotracheal intubation are at a higher risk of microbial infection by bacterial strains including nosocomial pneumonia. In a study of hospital patients undergoing oral intubation, it was found that gargling with $25 \mathrm{ml}(2.5 \mathrm{mg} / \mathrm{ml})$ of PVP-I for $1 \mathrm{~min}$, twice, reduced the presence of bacteria in the trachea [28]. Prior to intubation, all 19 patients in the control group (5 of whom had MRSA) who gargled tap water were found to have bacteria contaminating the tip of the tracheal tube upon removal. However, in the group that gargled PVP-I, general bacteria and MRSA were eradicated from the pharynx prior to intubation, as well as at the tip of the tracheal tube after removal. With proper use of PVP-I, surgical sites can be effectively decontaminated, and the risk of postoperative infection greatly reduced. Indeed, postoperative infection rates are lower when procedures include a sterilisation step with PVP-I [29]. Patients undergoing gastrointestinal procedures are at a higher risk of postoperative wound infection due to the difficulty in removing enteric bacteria present in the gut. However, PVP-I formulations have also been shown to be effective in preventing complications arising from infections in such settings [30], resulting in an average of five fewer days spent in hospital recovery. Additionally, the use of a PVP-I mouthwash prior to dental extraction procedures has been shown to reduce gingival bacteria, lowering the risk of bacteraemia [31].

Other sterilisation approaches can be considered for use in combination with PVP-I. Microbial pathogens such as influenza and tuberculosis can spread by airborne pathways in hospital settings. Although UVC light is known to inactivate such agents, even brief exposure to this highly energetic light can damage human tissue. In an interesting recent study, researchers used a UV-based sterilisation approach incorporating single-wavelength far-UVC light generated by filtered excilamps, which was found to kill pathogenic microorganisms [32]. Importantly, the wavelengths used cannot penetrate human skin or eye tissue, and are not powerful enough to cause biological damage to mammalian cells. Due to the considerably smaller scales at which microbial structures exist, the 222-nm far-UVC light was highly effective in inactivating the H1N1 strain of influenza A virus. The viral particles were suspended in aerosolised droplets, simulating those generated by human coughing and breathing. In addition to its affordability as an antiseptic approach, one major advantage of UVC light is that it is likely to be effective against all airborne microbes. Like PVP-I, this broad spectrum of effect is particularly important considering the multidrug-resistant variants of bacteria emerging in such settings.

\section{PVP-I FOR HYGIENIC INTERVENTIONS}

It has long been known that hand washing, when performed properly, can significantly reduce the carriage and spread of pathogens [33]. This has a direct effect on reducing patient morbidity and mortality from nosocomial infections. Hand washing is an important and established procedure for infection control with clinically-validated efficacy and a core component of protocols aimed at reducing infectious outbreaks [11]. The skin can act as a reservoir for infectious agents, and the use of PVP-I for hand disinfection represents an alternative to alcohol-based hand rubs, with medicated soaps containing PVP-I now readily available. Such PVP-I soaps have shown equivalent or superior efficacy to alcohol-based hand sanitisers when tested against norovirus, a common cause of gastroenteritis [20]. In contrast, chlorhexidine and triclosan-based hand washes, have been shown to be inferior against norovirus in practical application tests. Hand washing with PVPI-based formulations have shown similar 
antimicrobial efficacy to an alcohol-based hand rub, with both being preferable to the use of soap and water alone [34].

In a direct comparison of the virucidal activity of various hand sanitisers, including antimicrobial soaps and alcohol-based sanitisers, it was found in a modified fingerpad test that soap containing PVP-I was superior to the other soaps and sanitisers tested [35]. Three sanitisers were tested in the study, including ethanol, phosphoric acid, triclosan and chlorhexidine formulations. A modified test following the EN 1500 standard showed that the soap containing PVP-I was able to inactivate murine norovirus by approximately $4 \log _{10}$ steps. More recent hand-hygiene simulation studies have been described to test the comparative efficacy of different agents for hand antisepsis. In one study, volunteers prewashed their hands before artificially contaminating them with either Escherichia coli (for bactericidal testing) or murine norovirus (virucidal) [36]. Antisepsis agents were then used in a randomised approach and the number of test organisms released from the fingertips was calculated in terms of mean $\log _{10}$ reduction factor as per EN1499 guidelines. The direct comparison of PVP-I 7.5\% and chlorhexidine 4\% formulations showed a clear superiority of PVP-I against the murine norovirus, while both PVP-I and chlorhexidine were significantly better than soft soap against $E$. coli. Such experimental models could be helpful in broader assessments of antisepsis agents.

The Association for Professionals in Infection Control and Epidemiology (APIC) has published a series of guidelines for hand washing and hand antisepsis in healthcare settings to supplement those written by various other hygiene authorities, including the US Food and Drug Administration. The guidelines recommend formulations containing $7.5 \%$ iodine for use as a surgical hand scrub. Lower concentrations $(0.05 \%)$ have good antimicrobial activity due to the concentrations of free iodine increasing to some extent as the solution is diluted [37]. Thorough hand antisepsis, which can be achieved by hand washing or surgical scrubs with antimicrobial agents, is recommended in surgical settings before the performance of invasive procedures such as the placement of intravascular catheters, or in any scenario where it is deemed necessary to reduce numbers of resident skin flora and transient microorganisms on the skin.

With recent hospital reports of vancomycinresistant E. faecium emerging [1], as well as alcohol tolerance in some strains, it may be advisable to take new considerations into account. In cases where infectious enterococci are identified (independent of vancomycin resistance), hand disinfection is almost certainly recommendable $(30 \mathrm{~s})$. In cases where $E$. faecium has been confirmed, the extent to which hand disinfectants are used in the context of other available active substances should be considered. The disinfectants listed by the Disinfectants Commission of the Association for Applied Hygiene (VAH) also mentions products with various other active ingredients including $n$-propanols, peroxides, and iodine products. Combinations with quaternary compounds, phenol derivatives, guanidine derivatives or iodine-cleaving compounds can also be considered. For surface disinfection, approaches that rely solely on alcohol disinfection should be avoided and replaced with combination approaches with alcohol and other active substances. For final disinfection of patient rooms where E. faecium may be present, per compound-based preparations can be used.

PVP-I scrubs have better skin tolerance than soap formulations of chlorhexidine and quaternary ammonium compounds [38]. Although there is an urgent need for well-designed studies directly comparing the clinical and economic profiles of antiseptics in such settings, PVP-I can be considered the antiseptic of choice for the management of superficial skin infections.

Mouthwashes and gargles are commonly used in hospital settings to prevent respiratory and endotracheal infections. In one hospitalbased trial, adult subjects with chronic respiratory diseases and repeat infections gargled a PVP-I formulation multiple times daily for extended durations up to 2 years [39]. Prior to the initiation of the study, 14 patients experienced a total of 67 episodes of infections (with a mean of 4.8 episodes). This was reduced to a remarkable mean number of 2.0 following PVP-I 
gargling, representing a 58\% reduction in the number of episodes. The most common causative strains were identified as $H$. influenzae, $M$. catarrhalis and pneumococci, with both influenza and MRSA reducing by 50\%. Similarly, tests of PVP-I 7\% gargle/mouthwash diluted to reflect real-life scenarios (1:30 dilution; equivalent to a concentration of $0.23 \%$ PVP-I) according to EN13727 standards showed rapid antimicrobial effects after just $15 \mathrm{~s}$ of exposure [36]. Effective and clinically-meaningful bactericidal activity against Klebsiella pneumoniae and Streptococcus pneumoniae was observed under such conditions, as well as the inactivation of common agents of serious respiratory tract infections including SARS-CoV, MERSCoV, influenza virus A (H1N1) and rotavirus.

Seeking to investigate the efficacy of PVP-I gargle in non-hospital settings, a study of PVP-I, chlorhexidine gluconate (CHG), and cetylpiridium chloride (CPC) gargles was conducted, supported by a PVP-I study across eight middle schools in Japan [40]. PVP-I showed the highest bactericidal rate against all test strains observed within $30 \mathrm{~s}$ of exposure. Middle school students were trained and encouraged to use the gargles, with comparisons of absenteeism made between the schools that encouraged the practice and those that did not. In the middle school using the PVP-I gargle, absenteeism due to the common cold and influenza were significantly lower compared to the schools using the other two agents. The authors concluded that the use of PVP-I gargle resulted in a decrease in absenteeism due to cold and influenza infections.

\section{EFFICACY OF PVP-I IN COMPARISON TO OTHER ANTISEPTIC AGENTS}

It has been more than 60 years since PVP-I was first marketed as an antibiotic/antiseptic agent. Since its introduction, various other agents including triclosan and carbapenem have been introduced, although it has been 30 years since a new class of antibiotic was last discovered.

According to recent reviews, there have been no confirmed reports of resistance to PVP-I to date [41]. Numerous studies have shown that PVP-I has a broader antimicrobial spectrum than other available antiseptics including chloroxylenol, chlorhexidine, and quaternary ammonium compounds. Although alcoholbased antiseptics also have broad potency, unlike PVP-I formulations, they typically have no effect on fungal or bacterial spores. Interestingly, honey and maggots have been shown to have antibacterial properties when applied in therapeutic wound-treatment settings, although their potency in comparison to iodine against viruses and endospores remains to be determined [42].

\section{SAFETY AND TOLERABILITY}

PVP-I is well tolerated by the majority of patients, particularly when applied to the skin [9]. In comparison to chlorhexidine, for example, PVP-I has rarely been associated with allergic contact dermatitis, while urticarial or anaphylactic reactions have been exceedingly rare. The 2013 EU Safety Assessment Report Findings assessed human data involving $6.9 \mathrm{~g}$ of PVP-I applied to the hands and forearms for a contact time of less than $5 \mathrm{~min}$ and concluded that the proposed use of iodine in hand disinfection products is suitable for human health. Although PVP-I is generally seen as very safe, with long-term use, cases of thyroid dysfunction induced by PVP-I have been reported. For this reason, patients receiving PVP-I treatment for extended periods of time should be carefully monitored [43].

\section{CONCLUSIONS}

With recent reports of emerging resistance to antibiotics, including ampicillin and vancomycin, attention has turned to the use of broad-spectrum antiseptics in limiting hospitalbased infectious outbreaks. Despite its long history of efficacious use, no significant cases of resistance to iodine have emerged. This is thought to be due to its broad antimicrobial activity, which has been confirmed by global health authorities including the World Health 
Organization and the European Wound Management Association.

European guidelines for testing PVP-I formulations during hand washing and topical applications recommend a stepwise approach, combined with standardised tests using validated test models. Formulations like Betadine ${ }^{\circledR}$ antiseptic solution have demonstrated $>99.99 \%$ activity against both enveloped and non-enveloped viruses including Ebola, MERS, SARS coronavirus, influenza and HFMD viruses (Enterovirus 71 and Coxsackievirus A16). The potency and accessibility of PVP-I formulations is therefore likely to be of continuing and significant benefit for human health on a global scale, particularly in the developing world where ensuring affordable access to reliable antiseptic agents can be challenging.

Furthermore, in comparison to most antibiotics, the use of broad-spectrum antiseptics reduces the likelihood of resistance emerging due to multiple mechanisms of action targeting diverse aspects of cell biology and replication machinery. In contrast to PVP-I, bacterial resistance to chlorhexidine, quaternary ammonium salts, silver and triclosan has been documented.

The long track record of efficacious use of PVP-I in clinical settings is also an advantage for further clinical investigation. The WHO recommends decision-makers be guided by all available scientific evidence regarding the expected benefits and risks of any therapeutic application. For this reason, the considerable unmet medical needs that still remain for infectious diseases in home and healthcare environments call for the further investigation of PVP-I formulations in such settings.

\section{ACKNOWLEDGEMENTS}

Funding. Writing assistance and the Rapid Service Fees was funded by Mundipharma Singapore Holding Pte Limited, Singapore, who played no role in the study and collection, analysis, and interpretation of the data. All authors had full access to the articles reviewed in this manuscript and take complete responsibility for the integrity and accuracy of this manuscript.

Medical Writing and/or Editorial Assistance. Writing assistance was provided by Lee Farrand of Streamline Editing.

Authorship. All named authors meet the International Committee of Medical Journal Editors (ICMJE) criteria for authorship for this article, take responsibility for the integrity of the work as a whole, and have given their approval for this version to be published.

Authors' Contributions. The author conducted a review of the literature, provided directions in the writing of the manuscript, reviewed and approved the final manuscript.

Disclosures. Maren Eggers has been a consultant for Mundipharma Singapore Holding Pte Limited, and the scope of engagement included laboratory studies as well as scientific presentations in conferences, for which she received honoraria and travel expenses.

Compliance with Ethics Guidelines. This article is based on previously conducted studies and does not contain any studies with human participants or animals performed by any of the authors.

Open Access. This article is distributed under the terms of the Creative Commons Attribution-NonCommercial 4.0 International License (http://creativecommons.org/licenses/ by-nc/4.0/), which permits any noncommercial use, distribution, and reproduction in any medium, provided you give appropriate credit to the original author(s) and the source, provide a link to the Creative Commons license, and indicate if changes were made.

\section{REFERENCES}

1. Wassilew N, Seth-Smith HMB, Rolli E, et al. Outbreak of vancomycin-resistant Enterococcus faecium 
clone ST796, Switzerland, December 2017 to April 2018. Euro Surveill. 2018;23(29):1800351.

2. Rubinstein E, Keynan Y. Vancomycin-resistant enterococci. Crit Care Clin. 2013;29(4):841-52.

3. Prematunge C, MacDougall C, Johnstone J, Adomako K, Lam F, Robertson J, et al. VRE and VSE bacteremia outcomes in the era of effective VRE therapy: a systematic review and metaanalysis. Infect Control Hosp Epidemiol. 2016;37(1):26-35.

4. Bell JM, Turnidge JD, Coombs GW, Daley DA, Gottlieb T, Robson J, George N. Australian group on antimicrobial resistance Australian Enterobacteriaceae sepsis outcome programme annual report, 2014. Commun Dis Intell. 2016;40(2):E229-35.

5. Pidot SJ, Gao W, Buultjens AH, Monk IR, Guerillot $\mathrm{R}$, Carter GP, et al. Increasing tolerance of hospital Enterococcus faecium to handwash alcohols. Sci Transl Med. 2018;10:6115.

6. Gottrup F, Apelqvist J, Bjarnsholt T, Cooper R, Moore Z, Peters EJG, Probst S. EWMA document: antimicrobials and non-healing wounds: evidence, controversies and suggestions. J Wound Care. 2013;22(Sup5):S1-89.

7. Lachapelle JM, Castel O, Fueyo Casado A. Antiseptics in the era of bacterial resistance: a focus on povidone iodine. Future Med. 2013;10:579-92.

8. Pierard-Franchimont C, Paquet P, Arrese JE, Pierard GE. Healing rate and bacterial necrotizing vasculitis in venous leg ulcers. Dermatology. 1997;194:383-7.

9. Bigliardi PL, Alsagoff SAL, El-Kafrawi HY, et al. Povidone iodine in wound healing: a review of current concepts and practice. Int $\mathrm{J}$ Surg. 2017;44:260-8.

10. Eggers M, Eickmann M, Kowalski K, et al. Povidoneiodine hand wash and hand rub products demonstrated excellent in vitro virucidal efficacy against Ebola virus and modified vaccinia virus Ankara, the new European test virus for enveloped viruses. BMC Infect Dis. 2015;15:375.

11. Ripa S, Bruno R, Reder R. Clinical applications of Povidone-Iodine as a topical antimicrobial handbook of topical antimicrobials industrial applications industrial applications in consumer products and pharmaceuticals. Boca Raton: CRC; 2002.

12. AIHA Biohazards Committee. Biosafety-reference manual. 2nd ed. Akron: American Industrial Hygiene Association; 1995.

13. Miller B, et al. Laboratory safety: principles and practices. Washington, D.C.: American Society for Microbiology; 1986.
14. Eggers M, Koburger-Janssen T, Ward LS, Newby C, Müller S. Bactericidal and virucidal activity of Povidone-Iodine and chlorhexidine gluconate cleansers in an in vivo hand hygiene clinical simulation study. Infect Dis Ther. 2018;7(2):235-47.

15. Herruzo R, Vizcaino MJ, Yela R. Surgical hand preparation with chlorhexidine soap or povidone iodine: new methods to increase immediate and residual effectiveness, and provide a safe alternative to alcohol solutions. J Hosp Infect. 2018;98(4):365-8.

16. Schreier H, Erdos G, Reimer K, et al. Molecular effects of povidone-iodine on relevant microorganisms: an electron-microscopic and biochemical study. Dermatology. 1997;195(Suppl 2):111-6.

17. Mayer S, Boos M, Beyer A, Fluit AC, Schmitz FJ. Distribution of the antiseptic resistance genes qacA, qacB and qacC in 497 methicillin resistant and susceptible European isolates of Staphylococcus aureus. J Antimicrob Chemother. 2001;47(6):896-7.

18. Skurray RA, Rouch DA, Lyon BR, et al. Multiresistant Staphylococcus aureus: genetics and evolution of epidemic Australian strains. J Antimicrob Chemother. 1998;21(Suppl C):19-39.

19. Coia JE, Duckworth GJ, Edwards DI. Guidelines for the control and prevention of meticillin-resistant Staphylococcus aureus (MRSA) in healthcare facilities. J Hosp Infect. 2006;63S:S1eS44.

20. Kunisada T, Yamada K, Oda S, Hara O. Investigation on the efficacy of povidone-iodine against antiseptic-resistant species. Dermatology. 1997;195(Suppl 2):14-8.

21. Houang ET, Gilmore OJA, Reid C, et al. Absence of bacterial resistance to povidone iodine. J Clin Pathol. 1976;29:752-4.

22. Wand ME, Bock LJ, Bonney LC, Sutton JM. Mechanisms of increased resistance to chlorhexidine and cross-resistance to colistin following exposure of Klebsiella pneumoniae clinical isolates to chlorhexidine. Antimicrob Agents Chemother. 2017;61(1):e01162-16.

23. Boyce JM, Pittet D. Centers for Disease Control and Prevention. Guideline for hand hygiene in healthcare settings: recommendations of the Healthcare Infection Control Practices Advisory Committee and the HICPAC/SHEA/APIC/IDSA Hand Hygiene Task Force. Morbid Mortal Wkly Rep. 2002;51(RR16):1-45.

24. Eggers M, Koburger-Janssen T, Eickmann M, Zorn J. In vitro bactericidal and virucidal efficacy of Povidone-Iodine gargle/mouthwash against respiratory 
and oral tract pathogens. Infect Dis Ther. 2018;7:249-59.

25. World Health Organization. Influenza (seasonal). Fact sheet no 211. 2014. http://www.who.int/ mediacentre/factsheets/fs211/en/.

26. Sriwilaijaroen $\mathrm{N}$, Wilairat $\mathrm{P}$, Hiramatsu $\mathrm{H}$, et al. Mechanisms of the action of povidone-iodine against human and avian influenza A viruses: its effects on hemagglutination and sialidase activities. Virol J. 2009;6:124.

27. Kawana R, Kitamurd T, Nakagomi O, et al. Inactivation of human viruses by Povidone-lodine in comparison with other antiseptics. Dermatology. 1997; 195(suppl2):29-35.

28. Ogata Junichi, Minami Kouichiro, Miyamoto Hiroshi, Horishita Takafumi, Ogawa Midori, Sata Takeyoshi, Taniguchi Hatsumi. Gargling with povidone-iodine reduces the transport of bacteria during oral intubation. Can J Anesth. 2004;51(9):932-6.

29. Edwards R, Harding KG. Bacteria and wound healing. Curr Opin Infect Dis. 2004;17:91-6.

30. Paddle-Ledinek JE, Nasa Z, Cleland HJ. Effect of different wound dressings on cell viability and proliferation. Plast Reconstr Surg. 2006;117:110S-8S.

31. Boyce ST, Warden GD, Holder IA. Cytotoxicity testing of topical antimicrobial agents on human keratinocytes and fibroblasts for cultured skin grafts. J Burn Care Rehabil. 1995;16:97-103.

32. Welch D, Buonanno M, Grilj V, Shuryak I, Crickmore C, Bigelow AW, et al. Far-UVC light: a new tool to control the spread of airborne-mediated microbial diseases. Sci Rep. 2018;8:2752.

33. Larson E. APIC Guideline for handwashing and hand antisepsis in health care settings. Am J Infect Control. 1995;4:251-69.

34. Niedner R. Cytotoxicity and sensitization of povidone-iodine and other frequently used anti-infective agents. Dermatology. 1997;195(suppl2):89-92.
35. Steinmann J, Paulmann D, Becker B, Bischoff B, Steinmann E, Steinmann J. Comparison of virucidal activity of alcohol-based hand sanitizers versus antimicrobial hand soaps in vitro and in vivo. J Hosp Infect. 2012;82:277-80.

36. Eggers M, Koburger-Janssen T, Ward LS, Newby C, Muller S. Bactericidal and virucidal activity of povidone-iodine and chlorhexidine gluconate cleansers in an in vivo hand hygiene clinical simulation study. Infect Dis Ther. 2018. https://doi. org/10.1007/s40121-018-0202-5.

37. Larson EL, 1994 APIC Guidelines Committee. APIC guidelines for handwashing and hand antisepsis in health care settings. Am J Infect Control. 1995;23(4):251-69.

38. Lachapelle JM, Castel O, Casado AF, Leroy B, Micali G, Tennstedt D, Lambert J. Antiseptics in the era of bacterial resistance: a focus on povidone iodine. Clin Pract. 2013;10(5):579.

39. Nagatake T, Ahmed K, Oishi K. Prevention of respiratory infections by povidone-iodine gargle. Dermatology. 2002;204(suppl1):32-6.

40. Shiraishi T, Nakagawa Y. Evaluation of the bactericidal activity of povidone-iodine and commercially available gargle preparations. Dermatology. 2002;204(suppl 1):37-41.

41. Kramer A, Dissemond J, Kim S, Willy C, Mayer D, Papke R, Tuchmann F, Assadian O. Consensus on wound antisepsis: update 2018. Skin Pharmacol Physiol. 2018;31(1):28-58.

42. Cherniack EP. Bugs as drugs, Part 1: insects: the" new" alternative medicine for the 21st century. Altern Med Rev. 2010;15(2):124-35.

43. Nobukuni K, Hayakawa N, Namba R, Ihara Y, Sato $\mathrm{K}$, Takada H, Hayabara T, Kawahara S. The influence of long-term treatment with povidone-iodine on thyroid function. Dermatology. 1997;195(Suppl. 2):69-72. 